\title{
Rainwater tank sizing methodology development for Curitiba city
}

\section{Aldely Ângelo A. Teixeira ${ }^{1}$, Júlio Gomes ${ }^{2}$; Paulo Rodrigo da Costa Pacheco ${ }^{3}$,Yapur Dumit Gomez $^{4}$, Luiza Carla Girard T. Machado 5}

\author{
1,2 Universidade Positivo. Curitiba - PR. \\ 3,4,5 Universidade Federal do Pará (UFPA). Rua Augusto Corrêa No.1.Bairro: Guamá. Belém-PA. \\ Email:aldelyangelo@yahoo.com.br,jgomes@up.edu.br,rodrigoeng.costa@gmail.com,y_dumit_g@hotmail.com, \\ luiza.girard@gmail.com.br
}

Received: February 17th, 2017

Accepted: April 14th, 2017

Published: June $30^{\text {th }}, 2017$

Copyright $@ 2016$ by authors and Institute of Technology Galileo of Amazon (ITEGAM). This work is licensed under the Creative Commons Attribution International License (CC BY 4.0).

http://creativecommons.org/lic enses/by/4.0/ (c) (1) (3) Open Aecen

\begin{abstract}
The rainwater reservation is one of the most important units of the harvesting system by virtue of supplying the rain water demand at different times of the variation in consumption, especially the volume of rainwater entering the system is associated with annual precipitation. Thus determine the useful volume for use in potable use or non-potable activity is necessary. There are methods for rainwater tank sizing, some being established by standards in use and widely used designs, and other dimensions that are alternatives. The city of Curitiba and other Brazilian capitals are aware of laws which require new buildings over $500 \mathrm{~m}^{2}$ deployment of rainwater systems. The reservoir design seeks to present the useful volume necessary taking into account the rainfall and financial analysis of the implementation of these systems, which also takes into account the water consumption rates for public supply.

Keywords: Tank, Sizing, Curitiba.
\end{abstract}

\section{Desenvolvimento de metodologia de dimensionamento de reservatórios de águas pluviais para a cidade de Curitiba}

\begin{abstract}
RESUMO
A reservação de água de chuva representa uma das unidades mais importantes do sistema de aproveitamento, em virtude de suprir a demanda de água da chuva em diferentes momentos da variação de consumo, principalmente que o volume de água de chuva que entra no sistema está relacionado com a precipitação anual. Desta forma determinar o volume útil para consumo nas atividades de uso potáveis ou não é necessário. Existem métodos para dimensionamento de reservatório de água pluvial, sendo alguns estabelecidos por normas e bastante utilizados emprojetos de aproveitamento, e outros que são alternativas de dimensionamento. A cidade de Curitiba bemcomo outras capitais brasileiras estão atentas a legislações em que obrigam a novas construções acima de $500 \mathrm{~m}^{2}$ a implantação de sistemas de água da chuva. O dimensionamento de reservatório busca apresentar o volume útil necessário levando em conta a precipitação e análise financeira da implantação destes sistemas, que leva ainda em consideração as tarifas de consumo de água por abastecimento público.
\end{abstract}

Palavras Chaves: Reservatório, Dimensionamento, Curitiba.

\section{INTRODUÇÃO}

A água potável que chega a nossas casas é utilizada para todos os fins, tais como higiene, limpeza, alimentação, descargas sanitárias, irrigação, limpeza de veículos, pisos, calçadas, etc. Parte desta água necess ariamente não precisa ser tratada e poderia serde aproveitamento de água de chuva. Observando isto, a prefeitura municipal de Curitiba/PR vem exigindo de novas construções e reformas com áreas superiores a $500 \mathrm{~m}^{2}$ de área impermeável a implantação de sistemas de aproveitamento de águas pluviais para utilização em fins não potáveis.

Um aspecto importante relacionado à implantação de sistemas de aproveitamento de águas pluviais para utilização em fins não potáveis é o dimensionamento do reservatório para coleta e armazenamento do volume de água originado pela precipitação. 
Os métodos sugeridos pelas normas brasileiras são oriundos de técnicas aplicadas a grandes reservatórios, trabalhando, emgrande parte, em uma escala mensal de precipitação.

Além disso, não consideram a questão financeira no dimensionamento do reservatório de águas pluviais, o que é importante para o investidor privado e interessante em áreas urbanas, onde a falta do sistema tem por efeito apenas usar um volume menor de água tratada fornecida pela rede de abastecimento com o consequente ônus financeiro.

O presente estudo foi desenvolvido para facilitar a determinação do volume ótimo de reservatório, aliado ao custo de implantação do sistema de captação e a demanda de água para uso em fins não potáveis.

\section{MATERIAIS E MÉTODOS}

A NBR 15527 [1] prescreve as diretrizes para a implantação de sistemas para a captação e o aproveitamento da água da chuva captada de coberturas em áreas urbanas para fins não potáveis, salienta também que o volume do reservatório deve ser baseado em critérios técnicos, econômicos e ambientais, usufruindo-se de qualquer método contido na mesma ou outro, desde que seja justificado.

O método para dimensionamento de reservatório de águas pluviais foi desenvolvido a partir do método apresentado por [2], que propõe a definição do volume do reservatório baseado emuma análise financeira, a partir da comparação entre duas alternativas.

Alternativa 1: Empreendimento sem sistema de aproveitamento de águas pluviais.

Alternativa 2: Empreendimento com sistema de aproveitamento de águas pluviais.

O método proposto sugere a implantação de duas novas variáveis, denominadas de D' e V', que serão apresentadas a seguir. A variável D' é a relação entre a demanda para fins não potáveis e a área de captação, conforme Equação 2.

onde:

$$
D^{\prime}=\frac{D}{A c}
$$

$\mathrm{D}^{\prime}=$ demanda unitária diária $\left(\mathrm{L} / \mathrm{dia} / \mathrm{m}^{2}\right)$;

$\mathrm{D}=$ demanda diária (L/dia);

$\mathrm{Ac}=$ área de captação $\left(\mathrm{m}^{2}\right)$.

Já a variável V' é a relação entre o volume do reservatório e a área de captação, obedecendo a Equação 3.

$$
V^{\prime}=\frac{V_{R}}{A c}
$$

onde:

$\mathrm{V}^{\prime}=$ volume unitário $\left(\mathrm{m}^{3} / \mathrm{m}^{2}\right)$;

$\mathrm{VR}=$ volume do reservatório $\left(\mathrm{m}^{3}\right)$;

Ac $=$ área de captação $\left(\mathrm{m}^{2}\right)$.

Com is so obedecendo às restrições descritas pelo método proposto por [2], podemos reescreve-se a Equação 1 da seguinte forma:

$$
\frac{V_{t}}{A c}=\frac{V_{t-1}}{A c}+\left(\frac{V C_{t}}{A c}-D^{\prime}{ }_{t}\right)
$$

O método sugerido por [2] para a análise financeira é o método do valor presente, no qual valores futuros são descontados e trazidos para valores presentes, através do uso de uma taxa de desconto (i). Conforme descrito pelos autores a seguir.

Considerando-se o método do valor presente, as equações 4 e 5 apresentadas a seguir, são utilizadas respectivamente para a determinação dos custos relativos às Alternativas 1 e 2:

$$
C_{1}=\sum_{t=1}^{n} \frac{C u_{t} x \subset A_{t}}{(1+i / 10 O)^{t}}
$$

onde:

$\mathrm{C} 1$ = custo presente da Alternativa $1(\mathrm{R} \$)$;

Cut = custo unitário da água tratada no ano $\mathrm{t}(\mathrm{R} \$ / \mathrm{m} 3)$;

CAt $=$ consumo anual de água tratada no ano $\mathrm{t}$ (m3/ano);

$\mathrm{i}=$ taxa de desconto anual (\% ao ano);

$\mathrm{n}=$ horizonte do projeto (anos).

$\mathrm{C}_{2}=\mathrm{C}_{0}+\sum_{\mathrm{t}=1}^{\mathrm{n}} \frac{\mathrm{Cm}_{\mathrm{t}}+\mathrm{Cu}_{\mathrm{t}} \mathrm{x} \mathrm{CA}_{\mathrm{t}}}{(1+\mathrm{i} / 100)^{\mathrm{t}}}$

onde:

$\mathrm{C} 2$ = custo presente da Alternativa $2(\mathrm{R} \$)$;

$\mathrm{C} 0$ = custo de implantação do sistema (R\$);

$\mathrm{Cmt}=$ custo de manutenção do sistema no ano $\mathrm{t}(\mathrm{R} \$ / \mathrm{m} 3)$ e demais variáveis já definidas anteriormente.

A caracterização da chuva utilizada foi dada através do índice de precipitação, denominada também de altura pluviométrica. É um importante critério a utilização de períodos de observações longos, preferencialmente acima de 30 anos, para que se permita uma análise representativa da influência das condições climatológicas, bem como usufruir-se de dados de qualidade, ou seja, menor número de falhas. A característica de precipitação deste estudo tem como base a série de dados da estação pluviométrica de Curitiba. A Figura 1 apresenta a precipitação média anual do período de janeiro de 1976 a dezembro de 2009.

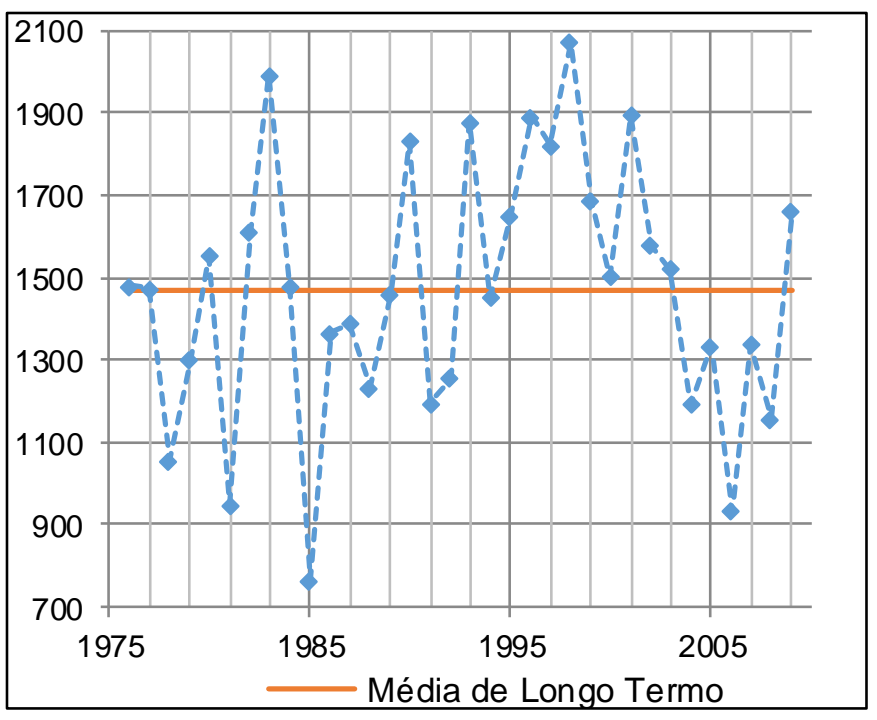

Figura 1: Precipitação média anual entre 1975-2005.

Fonte: Autores, (2016). 
Para a determinação do custo unitário da água tratada, foi utilizada a tabela de tarifas de saneamento básico da Companhia de Saneamento do Paraná [3] com ano de referência de 2012. Para o estudo não foi considerado o cálculo da tarifa social.

Foram estabelecidos 3 diferentes casos para exemplificação do método, procurou-se estabelecer cenários que caracterizassem distintos consumidores, diferentes áreas de captação bem como diferentes custos.

\section{RESULTADOS E DISCUSSÕES}

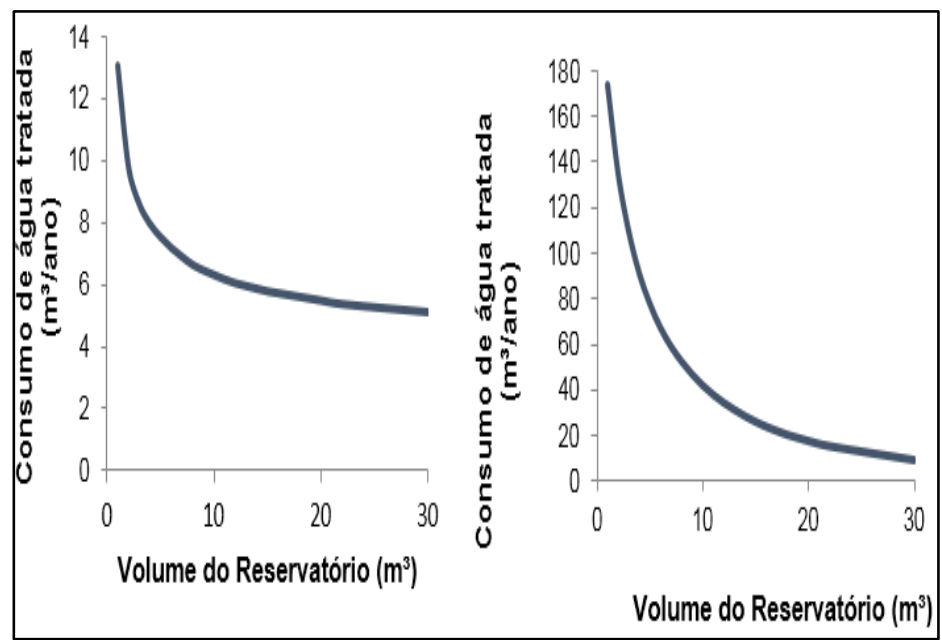

Figura 2: Curva relação Demanda x Volume do Reservatório - caso A e B.

Fonte: Autores, (2016).

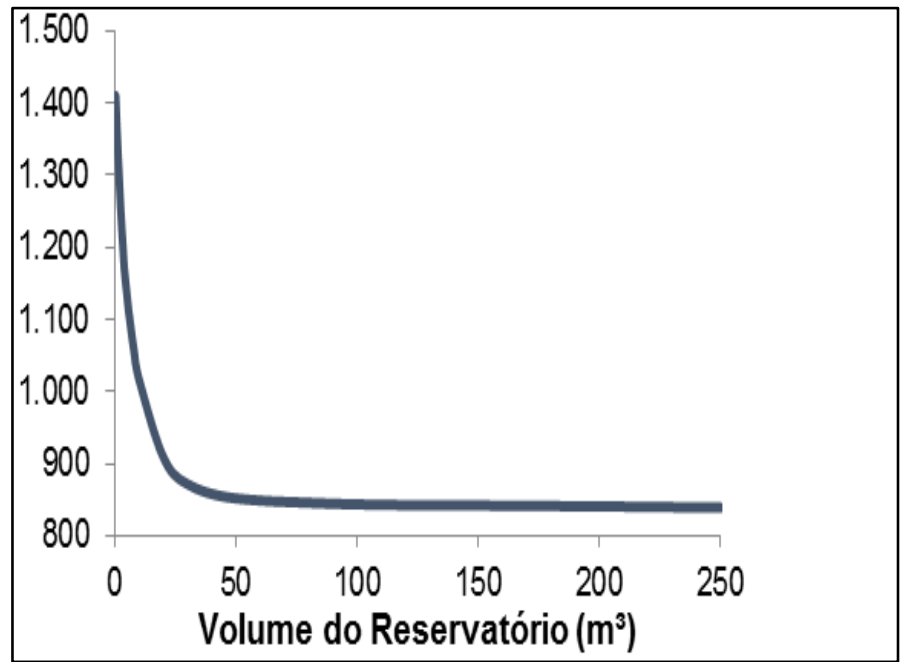

Figura 3: Curva relação Demanda x Volume do Reservatório Simulação 3.

Fonte: Autores, (2016).

Na Tabela 1 são apresentados os valores das variáveis necessárias para realizar o dimensionamento para cada estudo de caso. Para todos os casos foi utilizada uma taxa de desconto (i) igual a 5\% anual. Os custos de operação e manutenção foram estimados em 5\% anualmente do valor do custo de implantação. $\mathrm{O}$ horizonte do projeto (n) foi estabelecido em 20 anos.
Tabela 1: Condições iniciais para dimensionamento dos estudos de casos.

\begin{tabular}{|l|l|l|l|}
\hline Variável & Caso A & Caso B & Caso C \\
\hline $\mathrm{A}_{\mathrm{c}}\left(\mathrm{m}^{2}\right)$ & 30,00 & 450,00 & 600,00 \\
$\mathrm{D}(\mathrm{L} / \mathrm{dia})$ & 100,00 & 700,00 & 4000,00 \\
$\mathrm{C}_{0}\left(\mathrm{R} \$ / \mathrm{m}^{3}\right)$ & 500,00 & 500,00 & 1000,00 \\
$\mathrm{Cu}_{\mathrm{t}}\left(\mathrm{R} \$ / \mathrm{m}^{3}\right)$ & 2,00 & 2,00 & 4,50 \\
\hline
\end{tabular}

Fonte: Autores, (2016).

Caso A

O volume ótimo equivale a um reservatório de $0,20 \mathrm{~m}^{3}$. O consumo anual médio de água tratada para a alternativa 1 foi de $36,53 \mathrm{~m}^{3}$ e de $24,01 \mathrm{~m}^{3}$ para a alternativa 2 . O volume médio de água aproveitada anualmente foi de $12,52 \mathrm{~m}^{3}$, o que representa uma diminuição de $34,27 \%$ no consumo de água tratada.

O custo presente da alternativa 1 foi de $\mathrm{R} \$ 910,36$ e o da alternativa 2 foi de $\mathrm{R} \$ 760,62$ o que representa uma economia de R\$ 149,74 para o usuário com a implantação do sistema de aproveitamento de águas pluviais.

Caso B

O volume ótimo equivale a um reservatório de $1,94 \mathrm{~m}^{3}$. O consumo anual médio de água tratada para a alternativa 1 foi de $255,68 \mathrm{~m}^{3}$ e de $136,43 \mathrm{~m}^{3}$ para a alternativa 2 . O volume médio de água aproveitada anualmente foi de $119,25 \mathrm{~m}^{3}$, o que representa uma diminuição de 46, 64 \% no consumo de água tratada.

O custo presente da alternativa 1 foi de $\mathrm{R} \$ 6.372,55 \mathrm{e}$ o da alternativa 2 foi de $\mathrm{R} \$ 4.978,19$ o que representa uma economia de R\$ 1.394,36 para o usuário com a implantação do sistema de aproveitamento de águas pluviais.

Caso C

O volume ótimo equivale a um reservatório de $5,90 \mathrm{~m}^{3}$. O consumo anual médio de água tratada para a alternativa 1 foi de $1461,00 \mathrm{~m}^{3}$ e de $1110,08 \mathrm{~m}^{3}$ para a alternativa 2 . O volume médio de água aproveitada anualmente foi de $350,92 \mathrm{~m}^{3}$, o que representa uma diminuição de 24, $02 \%$ no consumo de água tratada.

$\mathrm{O}$ custo presente da alternativa 1 foi de $\mathrm{R} \$ 81.932,90$ e o da alternativa 2 foi de $\mathrm{R} \$ 71.836,04$ o que representa uma economia de R\$ $10.096,76$ para o usuário com a implantação do sistema de aproveitamento de águas pluviais.

\section{III.1 COMPARAÇÃO DE ESTUDOS DE CASOS}

Na Tabela 2 são apresentados os resultados das principais variáveis do dimensionamento por unidade de área de captação. Pode-se observar como a demanda unitária (D') tem uma relação direta com o volume unitário de armazenamento (V'). Observa-se uma relação direta entre D' e a economia no custo presente líquido (C') para os três casos. 
Tabela 2: Comparação dos casos por área unitária da superfície de captação.

\begin{tabular}{|l|l|l|l|}
\hline Variável & Caso A & Caso B & Caso C \\
\hline D' $\left(1 / \mathrm{m}^{2}\right)$ & 1,22 & 0,57 & 2,44 \\
V' $\left(1 / \mathrm{m}^{2}\right)$ & 6,67 & 4,32 & 9,84 \\
C' $\left(\mathrm{R} \$ / \mathrm{m}^{2}\right)$ & 4,99 & 3,10 & 16,83 \\
\hline
\end{tabular}

Fonte: Autores, (20160.

Se bem que os resultados apontam a que uma maior demanda por área de captação leva a uma maior economia de custos, deve-se considerar que outros fatores afetam o custo economizado. Na metodologia exposta o a diferencia em custos de implantação e custos de água tratada influenciam diretamente estes resultados.

\section{CONCLUSÕES}

O método proposto para o dimensionamento de reservatórios de armazenamento de águas pluviais é conceitualmente embasado no princípio de conservação de massa e é de fácil uso. Apresenta como característica exigir informações de obtenção relativamente simples, como a série de precipitações diárias, demanda diária, volume do reservatório e do custo de implantação, e permite que seja utilizado para diferentes faixas de consumo, de área de captação e custo de implantação.

Os resultados obtidos através dos exemplos mostram que quanto maior o reservatório, menor o consumo de água da rede pública de abastecimento, porém, a relação entre o consumo de água tratada e o volume de reservatório mostrou um comportamento assintótico, pois, o que determina o consumo mínimo de água tratada é a disponibilidade hídrica da região.

Portanto, o volume de captação é limitado, em outras palavras, não adianta aumentar o volume do reservatório se não há chuva o suficiente que possa enchê-lo. O comportamento as sintótico mostra que o consumo de água tratada tende ao mínimo, porém, ele será diferente de zero.

\section{REFERÊNCIAS}

[1] ABNT. "NBR 15527.Alternativas para a captação de água nas novas edificações. 2007.

ANA. 17 de Setembro de 2012. http://hidroweb.ana.gov.br (acesso em 17 de Setembro de 2012).

[2] GOMES, et al. Dimensionamento de reservatórios de águas pluviais, usando um critério financeiro. Revista Brasileira de Recursos Hídricos, março 2010.

[3] SANEPAR. 25 de junho de 2012. http://www.sanepar.com.br (acesso em 25 de junho de 2012).

[4] OURIQUES, RAFAEL ZINI, e LIDIANE BITENCOURT BARROSO. Água pluvial como alternativa para lavagem de veículos. HIDRO, n. 39 (janeiro 2010): 30 a 33.

[5] TOMAZ, PLÍNIO. Aproveitamento de Água de Chuva para Áreas Urbanas e Fins não Potáveis. Edição: Rubenal Hermano Santos. Vol. I. São Paulo, SP: Navegar Editora, 2003.

[6] SEEGER, L.M.K. Fficiência dos sistemas de aproveitamento de águas pluviais na região central do Rio Grande do Sul. 2008. 